\title{
Intrinsically disordered proteins as drug targets
}

\begin{abstract}
Intrinsically disordered proteins (IDPs) are characterized by a lack of folded structure. Since their identification more than a decade ago, they were designed as potential drug targets. However, nowadays, only few therapeutic molecules have been designed against them. Due to the nature of these proteins bioinformatics methods could have a key role disentangling IDPs related issues, which is key to design new therapeutic agents against them.
\end{abstract}

Keywords: intrinsically disordered proteins (Idps), small molecules, drug design, druggability, protein dynamics, protein flexibility, protein cavities, drug binding
Volume 5 Issue 2 - 2017

\author{
Alfons Nonell-Canals, Melchor Sanchez- \\ Martinez \\ Mind the Byte, Spain
}

Correspondence: Melchor Sanchez-Martinez, Mind the Byte, Parc Científic de Barcelona, c/Baldiri Reixac, 4-8, 08028 , Barcelona, Spain, Tel +34 934020938,

Email melchor@mindthebyte.com

Received: February 20, 2016 | Published: March 10, 2017

\section{Introduction}

\section{The challenge of targeting intrinsically disordered proteins}

Drug design is a long complex process. Translating basic research to the market (passing through drug discovery, preclinical and clinical studies) takes tens of years and costs billions of dollars. Computational techniques help to shorten the process economizing it. During the last three decades Computer-aided drug discovery/design methods (CAAD) have played a major role in the development of therapeutically important molecules. ${ }^{1,2}$ The importance of CADD techniques have been growing due to the drug discovery landscape has changed enormously getting complex.

Recent scientific and technological advances have allowed changing the traditional drug discovery paradigm, as the researchers has now access to myriads of high throughput biological data. ${ }^{3} \mathrm{CADD}$ approaches complement experimental screens guiding early-stage drug discovery efforts towards the so-called rational drug design. ${ }^{4}$

In rational drug design, all known theoretical and experimental knowledge of the system under study is used. Of great importance is the incorporation of the dynamical nature of the proteins. The structure-function paradigm must be substituted by the structure(dynamics)-function, as proteins are flexible entities, and thus move. Its function is closely related to flexibility and to carry out any function is necessary a conformational change. For instance, the determined conformations of a protein often differ in its ligandbound and -unbound forms. Protein conformational changes can open new ligand binding sites, whose exploration is key to fully assess the efficacy of a drug as well as to identify non-specific targeting with possible undesired effects. It is not only needed to block a certain conformation of a protein, it is necessary to modulate its dynamics. ${ }^{5-8}$ Targeting protein dynamics is a must.

A complex example where dynamics is crucial is constituted by Intrinsically Disordered Proteins (IDPs). ${ }^{9,10}$ For these proteins, their dynamic nature plays an essential role due to fundamentally exist as conformational ensembles. A similar example could be allosteric proteins for which protein flexibility have a great role been exploited to drug design. ${ }^{11,12}$

\section{Intrinsically disordered proteins}

IDPs are an emerging family of proteins whose most characteristic feature is that they don't present a folded structure, and thus challenge the sequence-structure-function paradigm. ${ }^{13-15}$ This lack of stable structure, over the entire protein length or in some regions (also named Intrinsically Disordered Regions or IDRs), provides them a structural plasticity which is not achievable by ordered proteins that is essential to carry out their cellular function. ${ }^{16,17}$ The paradigm should change to sequence-disorder-(dynamics)-function. ${ }^{17}$

Structural disorder is abundant in all species, although its level is higher in eukaryotes than in prokaryotes. IDPs (meaning long disordered regions of at least 30 residues) represent around the $30 \%$ of eukaryotic proteomes, and play key roles in a wide range of cellular processes, being involved in numerous human pathologies. ${ }^{15,16}$ Despite their importance, the IDPs study started at the end of the 20th century, and has been only in the last decade and a half when their existence has been widely accepted, becoming nowadays a hot scientific topic at both experimental and computational level.

Some regions of IDPs can adopt secondary structures, at least for a transient time, which is closely related to its function and to their interactions with binding partners. These structured elements can exist in the free protein, emerging upon conformational transitions or the combinations of both. ${ }^{18-20}$ The order-disordered transitions suffered by IDPs can be produced by several factors such as exogenous perturbations (for instance $\mathrm{pH}^{21-23} \mathrm{~T}^{\mathrm{a} 22,24-26}$ or macromolecular crowding) ${ }^{23,27-30}$ or binding (multiple) diverse molecular entities, such as proteins ${ }^{18,31,32}$ or small molecules. ${ }^{10,33-35}$ As they are very promiscuous molecules, IDPs can bind multiple partners. ${ }^{14}$

The conformational flexibility showed by IDPs is key to understand their behavior. There are growing evidences suggesting that when it is implicated in disease, is pathogenic causing so-called "conformational diseases" by means of misfolding, missignaling or even oligomerization processes. ${ }^{36-39}$ For instance, the conformational flexibility of the $\mathrm{N}$-terminal huntingtin is responsible of the formation of transient helical structures to Oligomerization. ${ }^{40,41}$ Targeting the conformational variability, i.e., targeting the dynamics of IDPs is a potential therapeutic strategy and in that sense, IDPs and their binding partners ${ }^{9,10,42}$ have been designed as targetable proteins.

\section{Drug design}

The widespread implication of IDPs in disease, make them perfect candidates to drug design, but unluckily, it is not happening and there are only few drugs targeting IDPs. This fact is a great contradiction 
as there are bioinformatics studies showing that Post Translational Modifications (PTMs) prefer disordered regions or that $79 \%$ of cancer associated proteins and $57 \%$ of the identified cardiovascular disease-associated proteins contain disordered regions of more than 30 residues. ${ }^{43,44}$ Compared with drug design pipe-lines for ordered proteins, for IDPs these processes remain in their infancy. ${ }^{44,45}$ Actually, the drugs related with IDPs consist on few molecules and some small peptides. ${ }^{10,42,44}$ This happens, among other reasons, because how IDPs perform their diverse function is not well understood. Thus, understand the functional and conformational properties of IDPs, is of great interest for a wide range of biological processes. Studying the structural determinants of this class of proteins could lead to understand their role in both healthy and altered-disease-associated cellular environments.

Unfortunately, traditional computational and experimental approaches have been hampered by diverse challenges. The most important is that IDPs present disordered regions that make impossible the use of crystallographic and cryo-electron microscopy methods as well as computational static methods such as rigid docking (over a single proteic conformation) because these techniques are not able to capture the conformational diversity of IDPs. IDPs exist in an ensemble of states, meaning that an ensemble of inter-converting conformers is required to describe the conformational behavior of IDPs.

The dynamic behavior of IDPs makes necessary the usage of "dynamical techniques" to their study. In this regard, important advances have been made towards IDPs understanding using spectroscopic techniques, such as Nuclear Magnetic Resonance (NMR). ${ }^{16,18,46}$ Small Angle X-ray Scattering ${ }^{47,48}$ or single-molecule fluorescence ${ }^{28,49-51}$ as well as employing atomistic and coarse-grained simulations..$^{20,52-55}$

By using the above-mentioned techniques some approximations have been reported to design drugs targeting IDPs. The common approaches are (I) directly bind to the disordered ensemble neutralizing the protein function/dysfunction and (II) bind to a binding partner and inhibit IDP binding or stabilize its bound state. ${ }^{10}$ They are aimed to targeting conformational transitions and regulatory elements besides of modulating Post Translational Modifications (PTMs). ${ }^{10}$ These strategies currently involved the development of small molecules and peptide inhibitors, but we cannot forget biologics. The design of monoclonal antibodies to capture IDPs plasticity could be also a great therapeutic opportunity to be further explored in the future. They are attractive therapeutic tools due to their high specificity and that they can be used to target protein-protein interactions. ${ }^{56}$ Besides, it has been shown that they are able to bind a peptide in different conformations. ${ }^{57}$ These findings suggest that could be possible to use them over multiconformational entities such as IDPs.

\section{Conformational landscape}

Conformational ensembles, also known as structural ensembles, are the accessible set of structures at a certain temperature describing the proteins structure. They are powerful tools to represent the range of conformations that can be sampled by proteins, thus allowing for an explicit representation of the dynamics of the protein. They are indicators of the structural heterogeneity of proteins. They can be generated purely experimentally, or theoretically for instance using NMR or Molecular Dynamics (MD) related techniques or by fitting (theoretical) ensembles to experimental data. ${ }^{58-61}$
Conformational ensembles have been employed to study different aspects related to fundamental properties of proteins, such as molecular recognition or protein folding and to study IDPs their usage is a widely-accepted option. IDPs are a perfect example of an scenario in that simulations and experiments have to converge to generate representative conformational ensembles, and a lot of efforts are put on it. ${ }^{62}$ The flexibility of proteins has been widely studied both experimentally and computationally. There exist a wide range of experimental techniques suitable to explore different time scales and resolutions. In IDPs the number of useful techniques is reduced because of their special characteristics. In that sense, computational methods are useful even more than in ordered proteins.

Experimental procedures provide a lot of useful data and computational approaches need it to carry out simulations as more realistic as possible. However, for IDPs, where dynamics is so important, computation clearly has advantages over experiments as it can describe dynamics completely and it can characterize the full conformational energy landscape of proteins (although conformational substrates and the rates of inter conversion can be located experimentally, the transition pathway at atomic resolution is out of reach. ${ }^{63}$ The computational simulations can be used as a 'virtual microscope' to study processes or molecules in a cell that are not directly accessible in experiments. ${ }^{64}$ Sometimes the synergies between experiments and computation are not so clear as for IDPs, and are ignored. However, in this cases it is strongly evidenced and in that sense the usage of CAAD techniques for IDPs related drug discovery have an enormous importance.

A useful method to generate conformational ensembles is classical MD. MD is a powerful technique to study protein motions generating dynamically relevant conformational ensembles, ${ }^{65}$ however there are scenarios in that it at cannot be routinely employed, as when the conformational space is too vast (as in IDPs) or the dynamics too slow. In these cases, enhanced sampling methods as Metadynamics ${ }^{66}$ or Replica Exchange Molecular Dynamics (REMD) ${ }^{67}$ as well as Coarse-Grained techniques could be used to overpass this limitation. There are also other option such as Brownian dynamics (BD) ${ }^{68}$ and Monte Carlo (MC) based methods for instance Replica exchange Monte Carlo (REMC) ${ }^{69,70}$ or Markov Chain Monte Carlo (MCMC), ${ }^{55}$ that have been employed to study IDPs with good results.

\section{Binding cavity detection and understanding}

Therapeutic ligands usually accomplish their mission by binding to small cavities (binding sites or pockets) of target proteins. IDPs are not an exception. The conformational landscape exploration upon binding is key to design drugs against IDPs. Identify the binding cavities over the generated ensembles is a must. In other words, the cavities must be detected for each ensemble and for each of the found pockets analyzing their structural and functional characteristics. To that extent, usual CAAD techniques can be useful to estimate the druggability and the effects of small molecules on IDP interactions. ${ }^{10,34,71,72}$ These methods analyze the cavities over static structures coming from experiments or simulations such as DrugPred, ${ }^{73}$ Cavity ${ }^{74}$ or fpocket, ${ }^{75}$ or coupled to MD simulations and calculate the druggability "on-the -fly", such as the recently developed JEDI. ${ }^{76}$

Zhang and coworkers ${ }^{44}$ have studied the ligand-binding cavities of diverse IDPs comparing some of their properties with those of ordered proteins. They concluded that IDPs are predicted to possess more binding cavities than ordered proteins of a similar length, showing 
different geometries. This agrees with previous studies which stated that IDPs possess greater surface and interface areas per residue than ordered proteins. Most importantly, they showed that the druggability of IDPs cavities may be comparable with those of ordered proteins. Besides they analyzed the conservation of the cavities along the different ensembles showing reasonable good results (higher than $50 \%)$.

The above-mentioned information is interesting but there are some issues that still should be addressed. For instance: IDPs binding sites can be targeted when they are completely disordered or only partially structured regions can host a ligand bound? There is a minimum percentage of ordered residues within the cavity necessary to drug binding? It is necessary that the binding pocket be embedded inside an ordered region or at least be flanked by ordered regions? , i.e., There are differences over IDPs and IDRs cavities? The structural characteristics of the binding pockets (size, volume, SASA, etc) rely on the disorder degree?

Fully understand IDPs binding processes will constitute the definitive step to rational design new drugs against them. In that sense the conformational landscape and the binding cavities exploration, upon ligand binding and in the unbound state, is the first step to reach that understanding.

\section{Conclusion}

IDPs seem to be perfect drug targets; however there is still a lack of knowledge as well as methodological gaps/flaws that difficult their usage. Nowadays there is quite clear how order to disorder transition and thus the adoption of secondary structure take place as well as its importance in disease. Moreover there are several techniques, or better said combinations of them, that constitute valid pipelines to their study and understanding.

However, direct targeting the conformational flexibility of IDPs is still in the very beginning. With a better understanding of the atomic details of the conformations of IDPs as well as the biophysical forces governing them, small molecules or even antibodies might be developed to stabilize distinct IDPs conformations or just to modulate IDPs interactions and thus their misfolding events.

There are still open structural and functional questions that should be addressed. More efforts should be done to address them, but we are in the correct way and attending to the great impact that IDPs have in human health we are assisting to crucial moments for our society. Be able to cope with disorder imply be able to fight against diseases ranging from cancer or neurodegenerative pathologies to orphan diseases, and computation has a lot to do on it. We reckon the actual and coming findings will bring IDPs the full recognition they deserve as drug targets, and hopefully several drugs will be designed in the next years targeting disorder and plasticity.

\section{Acknowledgements}

MSM thanks the Ministerio de Economia y Competitividad for a Torres Quevedo grant (PTQ-14-06579).

\section{Conflict of interest}

The author declares no conflict of interest.

\section{References}

1. Ou-Yang SS, Lu JY, Kong XQ, et al. Computational drug discovery. Acta Pharmacol Sin. 2012;33(9):1131-1140.
2. Sliwoski G, Kothiwale S, Meiler J, et al. Computational Methods in Drug Discovery. Pharmacol Rev. 2013;66(1):334-395.

3. Thibaut U. Bioinformatics and rational drug design. tools for discovery and better understanding of biological targets and mode of action of drugs. Scand J Gastroenterol Suppl. 2002;236:95-99.

4. Mavromoustakos T, Durdagi S, Koukoulitsa C, et al. Strategies in the rational drug design. Curr Med Chem. 2011;18(17):2517-2530.

5. Ferrari S, Costi, PM, Wade RC. Inhibitor Specificity via Protein Dynamics. Insights from the Design of Antibacterial Agents Targeted Against Thymidylate Synthase. Chem Biol. 2003;10(12):1183-1193.

6. Boehr DD, Nussinov R, Wright PE. The role of dynamic conformational ensembles in biomolecular recognition. Nat Chem Biol. 2009;5(11):789796.

7. Mandell DJ, Kortemme T. Computer-aided design of functional protein interactions. Nat Chem Biol. 2009;5(11):797-807.

8. Rebecca $\mathrm{C}$ Wade. Targeting protein dynamics in drug design. $J$ Cheminform. 2013;5(11):797-807.

9. Cheng Y, Le Gall T, Oldfield CJ, et al. Rational drug design via intrinsically disordered protein. Trends Biotechnol. 2006;24(10):435442.

10. Ambadipudi S, Zweckstetter M. Targeting intrinsically disordered proteins in rational drug discovery. Expert Opin Drug Discov. 2015;11(1):65-77.

11. Panjkovich A, Daura X. Exploiting protein flexibility to predict the location of allosteric sites. BMC Bioinformatics. 2012;25(13):273.

12. Wagner JR, Lee CT, Durrant JD, et al. Emerging Computational Methods for the Rational Discovery of Allosteric Drugs. Chem Rev. 2016;116(11):6370-6390.

13. Tompa P. Intrinsically disordered proteins. a 10-year recap. Trends Biochem Sci. 2012;37:509-516.

14. Uversky V N. Unusual biophysics of intrinsically disordered proteins Biochim Biophys Acta. 2013;1834(5):932-951.

15. Uversky VN. A decade and a half of protein intrinsic disorder. biology still waits for physics. Protein Sci. 2013;22(6):693-724.

16. Dyson HJ. Expanding the proteome. Disordered and alternatively folded proteins. Q Rev Biophys. 2011;44(4):467-518.

17. van der Lee R, Buljan M, Lang B, et al. Classification of Intrinsically Disordered Regions and Proteins. Chem Rev. 2014;114(13):6589-6631.

18. Wright PE, Dyson HJ. Linking folding and binding. Current Opinion in Structural Biology. 2009;19(1):31-38.

19. Uversky VN. Under-folded proteins: Conformational ensembles and their roles in protein folding, function and pathogenesis. Biopolymers. 2013;99(11):870-887.

20. Sancho D, De Baker CM, Best RB. In Computational Approaches to Protein Dyanmics. In: Fuxreiter M, editor. 2015:283-304.

21. Sugase K1, Dyson HJ, Wright PE. Mechanism of coupled folding and binding of an intrinsically disordered protein. Nature. 2007;447(7147):1021-1025.

22. Uversky VN. Intrinsically disordered proteins and their environment. Effects of strong denaturants, temperature, $\mathrm{pH}$, counter ions, membranes, binding partners, osmolytes, and macromolecular crowding. Protein $J$. 2009;28 (7-8):305-325.

23. Theillet FX, Binolfi A, Frembgen-Kesner T, et al. Physicochemical Properties of Cells and Their Effects on Intrinsically Disordered Proteins (IDPs). Chem Rev. 2014;114(13):6661-6714. 
24. Kjaergaard M, Nørholm AB, Hendus-Altenburger R, et al. Temperaturedependent structural changes in intrinsically disordered proteins: formation of alpha-helices or loss of polyproline II?. Protein Sci. 2010;19(8):1555-1564.

25. Rogers JM, Oleinikovas V, Shammas SL, et al. Interplay between partner and ligand facilitates the folding and binding of an intrinsically disordered protein. Proc Natl Acad Sci U S A. 2014;111(43):15420-15425.

26. Wuttke R, Hofmann H, Nettels D, et al. Temperature-dependent solvation modulates the dimensions of disordered proteins. Proc Natl Acad Sci US A. 2014;111(14):5213-5218.

27. Kuznetsova TG, Starodubtseva MN, Yegorenkov NI, et al. Atomic force microscopy probing of cell elasticity. Micron. 2007;38(8):824-833.

28. Soranno A, Koenig I, Borgia MB, et al. Single-molecule spectroscopy reveals polymer effects of disordered proteins in crowded environments. Proc Natl Acad Sci U S A. 2014;111(13):4735-4736.

29. Cino EA, Killoran RC, Karttunen M, et al. Binding of disordered proteins to a protein hub. Sci Rep. 2013;3:2305.

30. Qin S, Zhou HX. Effects of Macromolecular Crowding on the Conformational Ensembles of Disordered Proteins. J Phys Chem Lett. 2013:4(20)

31. Arai M, Sugase K, Dyson HJ, et al. Conformational propensities of intrinsically disordered proteins influence the mechanism of binding and folding. Proc Natl Acad Sci U S A. 2015;112(31):9614-9619.

32. Ciemny MP, Debinski A, Paczkowska M, et al. Mechanism of Folding and Binding of an Intrinsically Disordered Protein As Revealed by ab Initio Simulations. Journal of Chemical Theory and Computation. 2014;10:2224-2231.

33. Follis AV, Hammoudeh DI, Wang H, et al. Structural rationale for the coupled binding and unfolding of the c-Myconcoprotein by smal molecules. Chem Biol. 2008;15(11):1149-1155.

34. Michel J, Cuchillo R. The Impact of Small Molecule Binding on the Energy Landscape of the Intrinsically Disordered Protein C-Myc. PLoS One. 2012;7(7):1070.

35. Yu C, Niu X, Jin F, et al. Structure-based Inhibitor Design for the Intrinsically Disordered Protein c-Myc. Sci Rep. 2013;2(6):22298.

36. Vladimir N, Uversky. Wrecked regulation of intrinsically disordered proteins in diseases: pathogenicity of deregulated regulators. Front $\mathrm{Mol}$ Biosci. 2014;1(6):1-24.

37. Uversky VN. Intrinsically disordered proteins and their (disordered) proteomes in neurodegenerative disorders. Front Aging Neurosci. $2015 ; 2(7): 1-6$

38. Tóth G, Gardai SJ, Zago W, et al. Targeting the Intrinsically Disordered Structural Ensemble of $\alpha$-Synuclein by Small Molecules as a Potential Therapeutic Strategy for Parkinson's Disease. PLoS One. 2014;9(2):e87133.

39. Vladimir NUversky, Vrushank Davé, Lilia M Iakoucheva, tal. Pathological Unfoldomics of Uncontrolled Chaos: Intrinsically Disordered Proteins and Human Diseases. Chem Rev. 2014;114(13):6844-6879.

40. Michalik A1, Van Broeckhoven C. Pathogenesis of polyglutamine disorders: aggregation revisited. Hum Mol Genet. 2003;12(2):173-86

41. Długosz M, Trylska J. Secondary Structures of Native and Pathogenic Huntingtin N-Terminal Fragments. J Phys Chem B. 2011;115(40):1159711608 .

42. Tompa P. Unstructural biology coming of age. Curr Opin Struct Biol. 2011;21(3):419-25 (2011)

43. Oldfield C J, Xue B, Dunker A K, et al. Protein and Peptide Folding, Misfolding, and Non-Folding. Canada: John Wiley \& Sons; 2012. p. 239-277.
44. Yugang Zhang, Huaiqing Cao, Zhirong Liu. Binding cavities and druggability of intrinsically disordered proteins. Protein Sci. 2015;24(5):1-18

45. Fan Jin, Chen Yu, Luhua Lai, et al. Ligand Clouds around Protein Clouds: A Scenario of Ligand Binding with Intrinsically Disordered Proteins. PLoS Comput Biol. 2013;9(10):e1003249.

46. Ball KA, Phillips AH, Wemmer DE, et al. Differences in $\beta$ strand populations of monomeric $\mathrm{A} \beta 40$ and A $\beta 42$. Biophys $J$ 2013;104(12):2714-27124.

47. Kachala M, Valentini E, Svergun DI. Application of SAXS for the Structural Characterization of IDPs. Adv Exp Med Biol. 2015;870:261289.

48. Bernadó P, Svergun DI. Analysis of intrinsically disordered proteins by small-angle X-ray scattering. Methods Mol Biol. 2012;896:107-122.

49. Ferreon AC, Ferreon JC, Wright PE, et al. Modulation of allostery by protein intrinsic disorder. Nature. 2013;498(7454):390-394.

50. Schuler B, Hofmann H. Single-molecule spectroscopy of protein folding dynamics - expanding scope and timescales. Curr Opin Struct Biol. 2013;23(1):36-47.

51. Ferreon AC, Moran CR, Gambin Y, et al. Single-molecule fluorescence studies of intrinsically disordered proteins. Methods enzymol. 2010;472:179-204

52. Mao AH, Lyle N, Pappu RV. Describing sequence-ensemble relationships for intrinsically disordered proteins. Biochem J. 2013;449(2):307-318.

53. Lyle N, Das RK, Pappu RV. A quantitative measure for protein conformational heterogeneity. J Chem Phys. 2013;139(12):121907.

54. Fisher CK, Stultz CM. Protein structure along the order-disorder continuum. J Am Chem Soc. 2011;133(26):10022-10025.

55. Das RK, Pappu RV. Conformations of intrinsically disordered proteins are influenced by linear sequence distributions of oppositely charged residues. Proc Natl Acad Sci U S A. 2013;110(33):13392-13397.

56. Bakail M, Ochsenbein F. Targeting protein-protein interactions, a wide open field for drug design. Comptes Rendus Chim. 2016;19(1-2):19-27.

57. Chu HM, Wright J, Chan YH3, et al. Two potential therapeutic antibodies bind to a peptide segment of membrane-bound $\mathrm{IgE}$ in different conformations. Nat Commun. 2014;5:3139.

58. Vendruscolo M. Determination of conformationally heterogeneous states of proteins. Curr Opin Struct Biol. 2007;17(1):15-20.

59. Fenwick RB, Esteban-Martín S, Salvatella X. Understanding biomolecular motion, recognition, and allostery by use of conformational ensembles. Eur Biophys J. 2011;40(12):1339-1355.

60. Salvatella X. Protein Conformational Dynamics. Springer; 2014. p $67-85$.

61. Sanchez-Martinez M, Crehuet R. Application of the maximum entropy principle to determine ensembles of intrinsically disordered proteins from residual dipolar couplings. Phys Chem Chem Phys. 2014;16(47):26030 36039 .

62. Jensen MR, Ruigrok RW, Blackledge M. Describing intrinsically disordered proteins at atomic resolution by NMR. Curr Opin Struct Biol. 2013;23(3):426-435.

63. Henzler-Wildman KA, et al. Intrinsic motions along an enzymatic reaction trajectory. Nature. 2007;450:838-U13.

64. Hansmann. Multiscale Approaches to Protein Modelling. Springer; 2011. p. 209-230.

65. Showalter SA, Brüschweiler R. Quantitative Molecular Ensemble Interpretation of NMR Dipolar Couplings without Restraints. J Am Chem Soc. 2007;129(14):4158-4159. 
66. Zerze GH, Miller CM, Granata D, et al. Free Energy Surface of an Intrinsically Disordered Protein: Comparison between Temperature Replica Exchange Molecular Dynamics and Bias-Exchange Metadynamics. J Chem Theory Comput. 2015;11(6):2776-2782.

67. Chebaro Y, Ballard AJ, Chakraborty D, et al. Intrinsically Disordered Energy Landscapes. Sci Rep. 2015;5:10386.

68. Zhou HX, Pang X, Lu C. Rate constants and mechanisms of intrinsically disordered proteins binding to structured targets. Phys Chem Chem Phys. 2012;14(30):10466-10476.

69. Staneva I, Huang Y, Liu Z, et al. Binding of two intrinsically disordered peptides to a multi-specific protein: a combined Monte Carlo and molecular dynamics study. PLoS Comput Biol. 2012;8(9):e1002682.

70. Cong X, Casiraghi N, Rossetti G, et al. Role of Prion Disease-Linked Mutations in the Intrinsically Disordered N-terminal Domain of the Prion Protein. J Chem Theory Comput. 2013;9(11):5158-5167.

71. Cuchillo R, Michel J. Mechanisms of small-molecule binding to intrinsically disordered proteins. Biochem Soc Trans. 2012;40(5):1004 1008 .
72. Heller GT, Sormanni P, Vendruscolo M. Targeting disordered proteins with small molecules using entropy. Trends Biochem Sci. 2015;40(9):491-496.

73. Krasowski A, Muthas D, Sarkar A, et al. DrugPred: A Structure-Based Approach To Predict Protein Druggability Developed Using an Extensive Nonredundant Data Set. J Chem Inf Model. 2011;51(11):2829-2842.

74. Yuan Y, Pei J, Lai L. Binding site detection and druggability prediction of protein targets for structure-based drug design. Curr Pharm Des. 2013;19:2326-2333.

75. Le Guilloux V, Schmidtke P, Tuffery P. Fpocket: an open source platform for ligand pocket detection. BMC Bioinformatics. 2009;10:168.

76. Cuchillo R, Pinto-Gil K, Michel J. A Collective Variable for the Rapid Exploration of Protein Druggability. J Chem Theory Comput. 2015;11(3):1292-1307. 